\title{
ROTATIONAL DOPPLER BEAMING IN ECLIPSING BINARIES
}

\author{
PAUL J. GROOT ${ }^{1,2}$

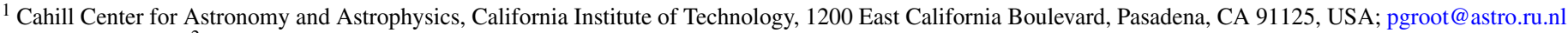 \\ ${ }^{2}$ Department of Astrophysics/IMAPP, Radboud University Nijmegen, P. O. Box 9010, 6500 GL Nijmegen, The Netherlands \\ Received 2011 April 18; accepted 2011 September 26; published 2011 December 29
}

\begin{abstract}
In eclipsing binaries the stellar rotation of the two components will cause a rotational Doppler beaming during eclipse ingress and egress when only part of the eclipsed component is covered. For eclipsing binaries with fast spinning components this photometric analog of the well-known spectroscopic Rossiter-McLaughlin effect can exceed the strength of the orbital effect. Example light curves are shown for a detached double white dwarf binary, a massive O-star binary and a transiting exoplanet case, similar to WASP-33b. Inclusion of the rotational Doppler beaming in eclipsing systems is a prerequisite for deriving the correct stellar parameters from fitting high-quality photometric light curves and can be used to determine stellar obliquities as well as, e.g., an independent measure of the rotational velocity in those systems that may be expected to be fully synchronized.
\end{abstract}

Key words: binaries: eclipsing - methods: observational - stars: rotation - techniques: photometric - white dwarfs

\section{INTRODUCTION}

Doppler beaming in stellar binaries was first discussed, in the context of stellar rotation, by Hills \& Dale (1974), after which it led a dormant life until it was rediscussed in the context of orbital motion by Maxted et al. (2000). The general theory was extensively discussed in Loeb \& Gaudi (2003) and Zucker et al. (2007) for stellar binaries and star-planet cases in the context of the orbital motion of the components in a binary. Because the flux density, $F_{v, 0}$, at a frequency $v$ is not a Lorentz-invariant quantity by itself, a brightening or dimming of the observed flux occurs depending on the radial velocity of the object.

In short, for non-relativistic velocities the beamed flux $F_{v}$ at frequency $v$ depends on the radial velocity $\left(v_{r}\right)$ and spectral slope $(\alpha)$ as (Equation (2) from Loeb \& Gaudi 2003):

$$
F_{\nu}=F_{v, 0}\left[1+(3-\alpha) \frac{v_{r}}{c}\right] \equiv F_{v, 0} B_{\alpha, v_{r}},
$$

where $F_{v, 0}$ is the unbeamed signal and $\alpha$ depends on the observing frequency and spectral slope of the object. Equation (1) defines the beaming factor $B_{\alpha, v_{r}}$. For blackbodies at a temperature $T_{\text {eff }}$ one can approximate $\alpha$ with Equation (3) from Loeb \& Gaudi (2003) or Zucker et al. (2007):

$$
\alpha(v)=\frac{e^{x}(3-x)-3}{e^{x}-1}=3-\frac{x e^{x}}{e^{x}-1},
$$

with $x \equiv h v / k T_{\text {eff }}$, and $h$ and $k$ Planck's and Boltzmann's constants, respectively.

Observationally, Doppler beaming has been detected in a small number of binaries so far. Van Kerkwijk et al. (2010) used the excellent photometric quality of the Kepler satellite to demonstrate the existence of Doppler beaming in two binary systems consisting of an A-type star and a white dwarf orbiting each other in 5.2 days and 23.9 days, respectively. Subsequently, the same effect has been found in a subdwarf B-white dwarf binary with a $9.6 \mathrm{hr}$ orbital period by Bloemen et al. (2010), also using Kepler data, in the transiting massive Jupiter/low-mass brown dwarf plus F3-type main-sequence star system CoroT-3b, with a 4.3 day orbital period by Mazeh \& Faigler (2010), and in the eclipsing, detached double white dwarf systems NLTT 11748 and SDSS J0651+38 in ground-based photometric data by Shporer et al. (2010) and Brown et al. (2011).
Apart from the Doppler beaming due to the orbital motion of the two stars in a binary, the same effect will also occur due to the rotational velocity of the stars. This was the original, but incomplete, context of Hills \& Dale (1974) and it has also been mentioned briefly by Van Kerkwijk et al. (2010). As the star spins on its rotational axis half of the star will be moving toward the observer and half of it will be moving away from the observer. In non-eclipse conditions and for axisymmetric stars the combined beaming effect of these two halves exactly cancels. However, during eclipse ingress and egress the partial coverage of the eclipsed component in the binary will break the symmetry and a net beaming will be the result. The amplitude of the beaming is set by the flux-weighted effect of the non-eclipsed part of the star and will be simulated in Section 3 for three cases. A fourth case, of solar-type binaries, has subsequently been discussed by Shporer et al. (2011).

In radial velocity studies, the related shift in the observed radial velocity of spectral lines during ingress and egress is well known as the Rossiter-McLaughlin effect, as first shown by Rossiter (1924) and McLaughlin (1924). The shape, amplitude, and asymmetry of the Rossiter-McLaughlin effect can be used to derive the projected rotational velocity of the star, prograde or retrograde rotation, and the obliquity of the binary orbit with respect to the stellar components. A detection of the rotational Doppler beaming (or photometric Rossiter-McLaughlin effect) may open up a photometric way of determining these same parameters in eclipsing binaries, substituting high spectral resolution studies with high signal-to-noise photometric time series. For very short period binaries such as the recently reported 12 minute orbital-period-detached white dwarf binary SDSS J0651+28 (Brown et al. 2011) the effect can, in principle, also be used for an independent determination of the spin-orbit coupling of the components to the orbit.

\section{ROTATIONAL DOPPLER BEAMING}

For a spherical star with radius $R$ the radial component $\left(v_{r}\right)$ of the rotational velocity $v_{\text {rot }}$ simply scales as

$$
v_{r}=v_{\text {rot }}\left(\frac{x}{R}\right) \sin i,
$$

with $x, y, z$ a right-handed coordinate system with $x, y$ defining the orbital plane and $z$ in the direction of orbital angular 
Table 1

System Parameters Used in the Simulations Shown in Figure 1

\begin{tabular}{lccc}
\hline \hline Parameter & Double Degenerate & O/B Star & A-star+Planet \\
\hline$M_{1}\left(M_{\odot}\right)$ & 0.43 & 80 & 1.495 \\
$M_{2}\left(M_{\odot}\right)$ & 0.17 & 20 & 0.00392 \\
$R_{1}\left(R_{\odot}\right)$ & 0.0148 & 14 & 1.44 \\
$R_{2}\left(R_{\odot}\right)$ & 0.0214 & 6.1 & 0.153 \\
$T_{1}(\mathrm{~K})$ & 10000 & 47800 & 6440 \\
$T_{2}(\mathrm{~K})$ & 16485 & 34200 & 1657 \\
$P_{\text {orb }}($ days $)$ & 0.0027 & 10.00 & 1.22 \\
$i($ deg $)$ & 90.0 & 90.0 & 90.0 \\
$K_{1}\left(\mathrm{~km} \mathrm{~s}^{-1}\right)$ & 218.8 & 9.2 & 0.5 \\
$K_{2}\left(\mathrm{~km} \mathrm{~s}^{-1}\right)$ & 553.5 & 36.7 & 185.9 \\
$v_{\text {rot }, 1}\left(\mathrm{~km} \mathrm{~s}^{-1}\right)$ & 1148.8 and 27.7 & 510.0 and 70.9 & 217.4 and 86.4 \\
$v_{\text {rot }, 2}\left(\mathrm{~km} \mathrm{~s}^{-1}\right)$ & 601.3 and 40.0 & 386.3 and 30.9 & 34.1 and 6.4 \\
$\alpha_{1}$ & 0.36 & 1.72 & -0.81 \\
$\alpha_{2}$ & 1.10 & 1.61 & -11.47 \\
\hline
\end{tabular}

momentum for an edge-on system. In our sign convention, prograde rotation of the stars with respect to the orbit results in positive rotational velocities. The orbital inclination with respect to the plane of the sky, $i$, is defined, as usual, to be $0^{\circ}$ for a face-on orbit and $90^{\circ}$ for an edge-on orbit.

The maximum rotational velocity of a star is set by its breakup velocity, $v_{\text {break }}=\sqrt{\frac{M G}{R}}$, and its minimum velocity is in principle unbounded, but, in short-period systems where tidal forces are strong enough to synchronize the system, will be set by the synchronization velocity $v_{\mathrm{sync}}=\frac{2 \pi R}{P_{\text {orb }}}$. In wide binaries where tidal synchronization is ineffective, there is no hard lower limit to the rotational velocity of each of the components. In these equations, $M$ is the mass of one of the components of the binary, $R$ is the radius of this component, $P_{\text {orb }}$ is the binary orbital period, and $G$ is Newton's gravitational constant. For main-sequence stars maximum rotational velocities are reached for early-type stars and can exceed $300 \mathrm{~km} \mathrm{~s}^{-1}$. After the onset of the magnetic dynamo around spectral type F1V, when stars develop a convective outer layer, the rotational velocity drops very quickly due to magnetic braking (see, e.g., Groot et al. 1996). In white dwarfs very high rotational velocities can be reached. Using the Eggleton mass-radius relation for fully degenerate white dwarfs as reported by Verbunt \& Rappaport (1988), a $1.2 M_{\odot}$ white dwarf can have a break-up velocity of $>6000 \mathrm{~km} \mathrm{~s}^{-1}$.

\section{SIMULATIONS}

To illustrate the rotational beaming effect we used Kepler's laws, the white dwarf mass-radius relation mentioned above, a massive star main-sequence mass-radius relation (at solar metallicity) from Pols et al. (1998), stars with no limb darkening (but, see Section 3.1), and Equations (1) and (2). The two stars are denoted by $M_{1}$ and $M_{2}$ with $M_{1}$ the more massive object, and the mass ratio $q=M_{2} / M_{1}<1$. Each star is given a blackbody spectrum characterized by the effective temperature $T_{\text {eff }}$. The orbital Doppler beaming has been included in the simulations, but, for clarity and simplicity, additional effects such as ellipsoidal variations, the reflection effect, and gravitational lensing have not been included. For illustration purposes all binaries are assumed to be seen exactly edgeon $\left(i=90^{\circ}\right)$. Three types of binaries have been simulated and an overview of system parameters is given in Table 1: a detached white-dwarf-white-dwarf binary similar to the one recently reported by Kilic et al. (2011) at an orbital period of 39 minutes; a massive O-star binary consisting of two main-sequence stars of $20 M_{\odot}$ and $80 M_{\odot}$ in a 10 day period orbit; and a transiting exoplanet around a fast rotating A-type star, similar to the WASP-33b system (Collier Cameron et al. 2010). For each system the effect is shown for an effective wavelength of $\lambda=6000 \AA$, which, together with the effective temperature, determines the $\alpha$ factor in Equation (2). In all three systems the rotational velocity of both components is varied between break-up and synchronization. For the WASP33 b-like exoplanet case, the measured rotational velocity of the A-star $\left(v_{\text {rot }} \sin i=86.5 \mathrm{~km} \mathrm{~s}^{-1}\right)$ was also simulated. The orbital phase $\phi$ is defined with respect to superior conjunction of the secondary. The projected surface area of the components has been divided in a $2000 \times 2000$ grid with dimensions of $2 R \times 2 R$, where positive values for the flux and rotational velocity were assigned for all values within a radius equal to $R$, and all points outside $R$ were set to zero. Fluxes were assigned as relative to the other component in the binary, with the flux of the secondary component set to unity. The position of the two components along the orbit was calculated for 4000 phase bins. At each phase bin the projected areal overlap between the two components has been calculated and the flux from the eclipsed component adjusted accordingly. A flux-weighted mean rotational velocity of the star is calculated and checked to be consistent with zero (within the numerical noise and finite grid width) for all phases outside of the primary or secondary eclipse.

Figure 1 shows the results for the three binaries in each of the three panels. The top panels show the total Doppler beaming factor $B_{\alpha, v_{r}}$ for both components in the binary. For clarity, both the orbital effect (the slow sinusoidal variation with a period equal to the orbital period) and the rotational effect (the excursions on top of the orbital variations during eclipse ingress and egress) are shown. In each figure the thick line indicates both components rotating at $v_{\text {rot }}=0.5 v_{\text {break }}$ and the thin line has both components rotating at $v_{\text {rot }}=v_{\text {sync }}$ with the exception of the panel on the transiting planet, where the star has been set to rotate at the measured rotational velocity of $v_{\text {rot }}=86.5 \mathrm{~km} \mathrm{~s}^{-1}$. Figure 1 shows that for substantial rotational velocity of the luminous component in the binary, the rotational Doppler beaming effect can be as large or larger than the orbital variation, similar to the spectroscopic Rossiter-McLaughlin effect (Gaudi \& Winn 2007). It is also clear from Figure 1 that a correct inclusion of the rotational Doppler beaming is a prerequisite to derive the correct parameters of the components in the binary from eclipse light curve fitting when very high quality data on a promising system is available.

\subsection{Obliquity and Limb Darkening}

The shape and amplitude of the rotational Doppler beaming effect not only depend on the rotational velocity, various other effects will play a role in determining the exact shape: the inclination of the orbit and the obliquity of the rotational axis, oblateness and axial symmetry of the stars, limb darkening and/or limb brightening, and differential rotation. In noneclipsing, but highly distorted systems, where, e.g., one of the components is (nearly) filling its Roche lobe and is for instance irradiated asymmetrically by an accretion disk hot spot, the asymmetric distribution of light on the distorted star can even lead to ellipsoidal terms on the orbital signal. For the moment, however, these highly distorted systems will not be considered and only an illustration of the obliquity and limb darkening will be given. 


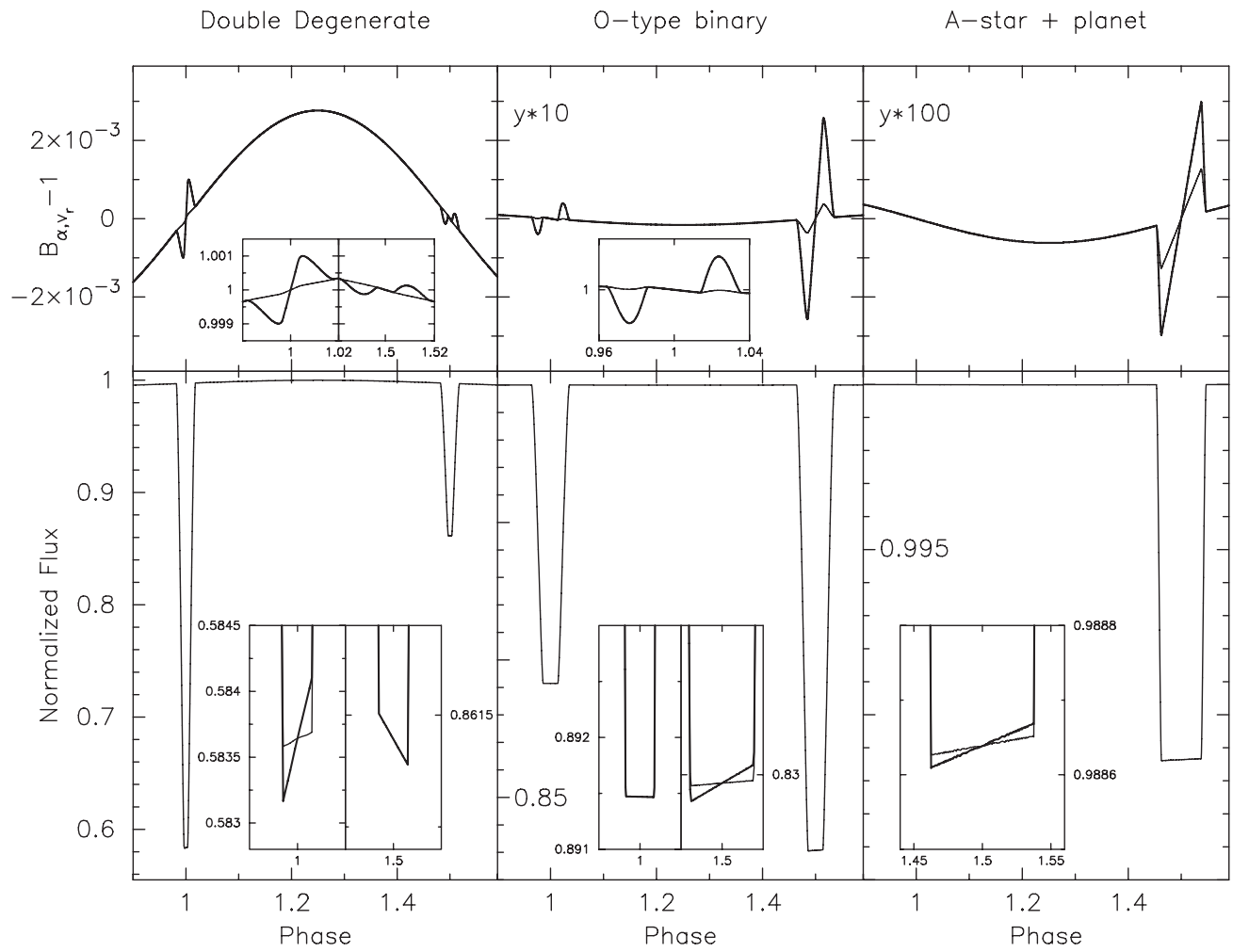

Figure 1. Simulated eclipses (bottom) and the photometric Doppler beaming factor $\left(B_{\alpha, v_{r}}\right.$, top) for three eclipsing binary systems: a detached double degenerate system (left), a massive O-type binary (middle), and a planet around an A-star (right). Insets show close-ups of the light curve and beaming profile during the eclipses. Thick lines are for $v_{\text {rot }}=0.5 v_{\text {break }}$ and thin lines are for $v_{\text {rot }}=v_{\text {sync }}$, except for the star-planet case where the thin line is for $v_{\text {rot }}=86.5 \mathrm{~km} \mathrm{~s}{ }^{-1}$.

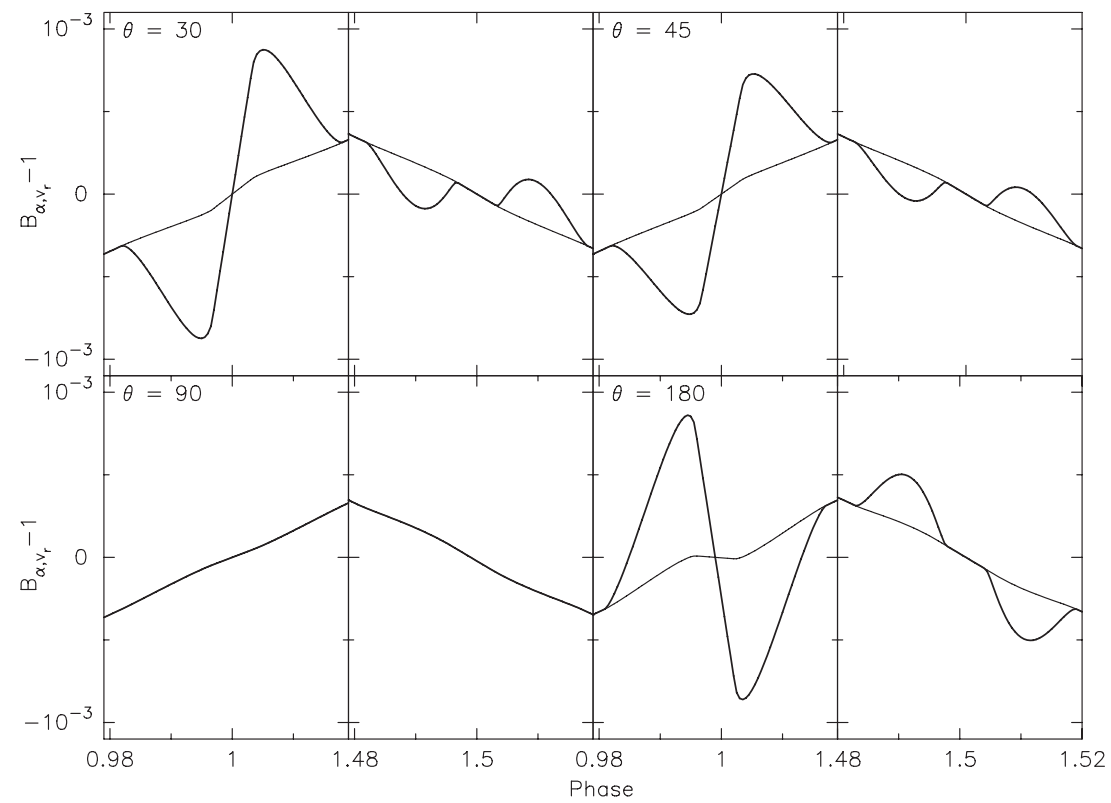

Figure 2. Beaming factors for a detached double degenerate system as in Figure 1, but now with both components obliquely rotating with respect to the orbital plane at an angle $\theta$, as indicated in the four subpanels.

The same double degenerate binary as in Section 3 is taken, but now the obliqueness, $\theta$, of the rotational axis of the two stars with respect to the orbital plane is varied between $\theta=30^{\circ}, 45^{\circ}$, $90^{\circ}$ (oblique rotator), and $180^{\circ}$ (retrograde rotator). As expected, the effect of obliqueness of the rotational axis is to decrease and eventually reverse the amplitude of the beaming (Figure 2). If a star is precessing in a binary this will present itself as a changing obliqueness with time. An observational detection through the rotational Doppler beaming would allow, e.g., a determination of the spin-orbit angular momentum coupling in close binaries and/or hierarchical triples.

Limb darkening is a fundamental property of stellar atmospheres which is reasonably well understood in mainsequence stars, but not so much in white dwarf atmospheres. 


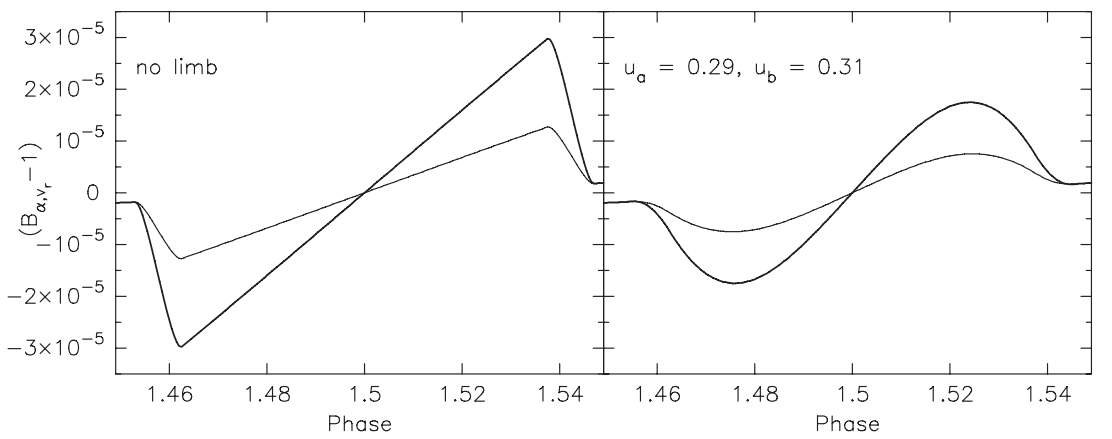

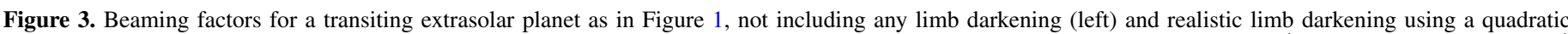

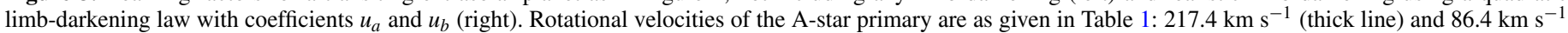
(thin line).

In helium-dominated, high-density white dwarf atmospheres limb darkening could even be largely absent due to internal refraction within the atmosphere (Kowalski \& Saumon 2004).

Using a quadratic limb-darkening law,

$$
F_{v}(\mu)=F_{0}\left[1-u_{a}(1-\mu)-u_{b}(1-\mu)^{2}\right],
$$

with $\mu=\cos \xi$ and $\xi$ the angle between the normal to the surface and the line of sight of the observer to describe the limb darkening; Figure 3 shows the effect for the transiting planet case (Figure 1), for limb-darkening coefficients of $u_{a}=$ 0.29 and $u_{b}=0.31$, appropriate for a $T_{\text {eff }}=6440 \mathrm{~K}$ star in the Kepler passband (Sing 2010). The general effect of limb darkening is to "soften" the edges of a star, from which it is conceptually easy to deduce that it will impact the height of the rotational Doppler beaming, in the same way that it affects the Rossiter-McLaughlin effect. The contribution to the total light from the most extremely beamed parts of the stars is lessened for a positive limb darkening. Figure 3 shows that indeed not only the amplitude of the Doppler beaming is suppressed, but also the skewness of the profile is significantly reduced. It is clear from this figure that a realistic modeling of transiting planet light curves where the Doppler beaming is included needs to include a limb-darkening term as well. However, if indeed, in highdensity, helium-dominated white dwarf atmospheres the limbdarkening coefficient is close to zero, the resulting rotational Doppler beaming should hardly be affected and may still show the sharp edges displayed in Figure 1.

\section{DETECTABILITY}

The ultimate goal of these investigations would of course be to first detect and subsequently use the rotational Doppler beaming to determine and constrain stellar and binary parameters. As can be seen from Figure 1, even in the best of cases, such as the double degenerates, this detection is going to be challenging. The fractional amplitude of the rotational beaming varies between $10^{-3} \lesssim B_{\alpha, v_{r}} \lesssim 10^{-6}$. To firmly establish the shape and amplitude the photometric accuracy needed would be a factor 10 smaller than this amplitude at least. The Kepler light curves show precisions in the $10^{-4}-10^{-5}$ range, and it should therefore be possible to detect the effect. In fact, a close look at Figure 3 in Bloemen et al. (2010) appears to show a residual effect in the primary eclipse profile by comparing their "R.+E.+L.+Beaming" panel with the inset in the left panel of Figure 1 in Section 3. However, this "by eye" residual does not clearly show up in the "Residuals" panel in Figure 3 in Bloemen et al. (2010).
In general, the detectability is a combination of source flux, eclipse duration, instrument stability, and intrinsic amplitude. Source flux can be optimized using a bigger telescope, eclipse duration is unfortunately a given for an individual source, instrument stability is in the design of the instrument and tends to be highest for space missions, even though modern photometers on the ground now achieve Kepler-like precision (e.g., de Mooij et al. 2011). For a more formal derivation of detectability I refer to Shporer et al. (2011), but the recently detected, eclipsing 12 minute orbital period binary SDSS J0651+28 (Brown et al. 2011) makes an illustrative example. Using the stellar parameters listed in Brown et al. (2011) and assuming a fully edge-on, aligned, and synchronized system (but see Piro 2011), the fractional amplitude of the Doppler beaming would be $1.3 \times 10^{-3}$. The best way to determine the rotational beaming signal is to determine the light-curve shape during primary mideclipse (inset of Figure 1), which for SDSS J0651+28 lasts for $\sim 0.05 P_{\text {orb }}$. As a rule of thumb I will use a minimum of 10 samples per eclipse and a photometric precision of a tenth of the amplitude to be a minimum for a positive detection of the effect, i.e., for SDSS J0651+28 a precision of $10^{-4}$ in the photometry needs to be obtained in $4 \mathrm{~s}$ on a $g=19.06$ magnitude object. Assuming only Poisson statistics as a noise contributor one therefore needs to collect $10^{8}$ photons per $4 \mathrm{~s}$ interval, requiring the equivalent of a $180 \mathrm{~m}$ telescope, even far out of reach in the Extremely Large Telescope (ELT) era. ${ }^{3}$ Of course, assuming perfect stability in the source, one can stack an arbitrary number of orbits to achieve the same precision. Even on a $10 \mathrm{~m}$ class telescope this would require the stacking of more than a thousand orbits, which is unfeasible in the short run, unless we find a system that is 5-6 mag brighter in the sky than SDSS J0651+28.

A much more promising case is presented by the O-type binaries, where the orbital period is much longer (10 days in our example), and, although mid-eclipse lasts for a shorter part of the orbital period, roughly $0.025 P_{\text {orb }}$, the "tenth of mid-eclipse" requirement translates into a duration of 36 minutes during which to collect the signal. The fractional amplitude is now only $10^{-4}$, requiring a precision at the $10^{-5}$ level, but Kepler has shown this to be feasible. We now need to collect the photons at a rate of $4.5 \times 10^{6} \mathrm{~s}^{-1}$. If our hypothetical binary were to be $g=14.0$, this is achievable on a $4 \mathrm{~m}$ class telescope by stacking a modest number of orbits (order 10), again assuming perfect source and instrument stability. In practice, of course,

\footnotetext{
3 Here I have used a photon flux of $10^{6}$ photons $\mathrm{s}^{-1} \mathrm{~cm}^{-2}$ for a $g=0$ object at the top of Earth's atmosphere.
} 
atmospheric and electronic (detector) stability may very well be the limiting factor.

\section{DISCUSSION AND CONCLUSION}

For very high quality data on eclipsing systems, inclusion of the effect of rotational Doppler beaming in light curve fitting procedures is necessary to derive the correct parameters of the binary. Since Doppler beaming is a geometrical effect, not including it will lead to a systematic error in the parameter derivation. The sign and magnitude of the error depends very much on the system properties of the binary. In prograde rotating binaries the extra "dimming" of the binary at ingress and the extra "brightening" during egress will cause an asymmetric eclipse profile that will skew a timing solution to a mid-eclipse phase that is too early. For retrograde rotation the effect on the timing will be the opposite.

From the simulations shown here it is clear that the detection of rotational Doppler beaming should be a mere matter of time and depends on the availability of suitable systems more than on the accuracy of current photometric systems. The most promising case would be a non-synchronously rotating eclipsing double degenerate system, if it is bright enough $(V<15)$. Currently none of these are known. Observationally there is a strong bias to find eclipsing double degenerates in short-period systems where the ratio of the white dwarf radius over the orbital separation is smallest and the system will be observed to be eclipsing over a wider range of inclinations. Unfortunately, short-period double-detached binaries also have the highest probability of (nearly) synchronous rotation since they are the product of two episodes of common-envelope evolution (e.g., Nelemans et al. 2001). This assumption does, however, depend strongly on the rotational coupling of the core of a red giant to its outer envelope (Sweigart \& Mengel 1979), and on the timescale of the common-envelope phase itself compared to the synchronization timescale (see, e.g., Taam \& Sandquist 2000). Since the common-envelope phase is expected to be relatively short, a decoupled core may remain unsynchronized. Prime targets should therefore be young, hot white dwarfs, or even cores of planetary nuclei, in short-period systems with another white dwarf, i.e., just after the "birth" of the second white dwarf in the system. It is unfortunate that the central binaries in the nova-planetary nebula system V458 Vul (Wesson et al. 2008; Rodríguez-Gil et al. 2010) and the planetary nebula system Hen 2-248 (Santander-García et al. 2011) do not appear to be eclipsing as these would have been almost ideal systems. The eclipsing double degenerate system NLT 11448 (Steinfadt et al.
2010) is also an obvious candidate for detection of rotational Doppler beaming. Although promising at first we have shown that the faintness and short period of SDSS J0651+28 makes it a much harder target.

Although the expected effect is a factor 10 smaller than in double degenerate systems, eclipsing O/B stars are perhaps an even more promising class of candidates. In general, the orbital periods will be much longer (days instead of hours), which causes the eclipse ingress and egress to last much longer, and therefore also allows for the accumulation of very high signal-tonoise ratio data. Since accurate photometry requires the presence of many comparison stars to obtain high-precision differential light curves, slightly fainter, far-away, and/or reddened $\mathrm{O} / \mathrm{B}$ binaries in the magnitude range $10<V<15$ may be more attractive candidates than very bright $(V<10)$ nearby systems.

\section{REFERENCES}

Bloemen, S., Marsh, T. R., Østensen, R. H., et al. 2010, MNRAS, 410, 1787 Brown, W. R., Kilic, M., Hermes, J. J., et al. 2011, ApJ, 737, L23

Collier Cameron, A., Günther, E., Smalley, B., et al. 2010, MNRAS, 407, 507 de Mooij, E. J. W., de Kok, R. J., Nefs, S. V., \& Snellen, I. A. G. 2011, A\&A, 528,49

Gaudi, B. S., \& Winn, J. N. 2007, ApJ, 655, 550

Groot, P. J., Piters, A. M., \& Van Paradijs, J. 1996, A\&AS, 118, 545

Hills, J. G., \& Dale, T. M. 1974, A\&A, 30, 135

Kilic, M., Brown, W. R., Kenyon, S. J., et al. 2011, MNRAS, 413, L101

Kowalski, P. M., \& Saumon, D. 2004, ApJ, 607, 970

Loeb, A., \& Gaudi, B. S. 2003, ApJ, 588, 117

Maxted, P. F. L., Marsh, T. R., \& North, R. C. 2000, MNRAS, 317, L41

Mazeh, T., \& Faigler, S. 2010, A\&A, 521, L59

McLaughlin, D. B. 1924, ApJ, 60, 22

Nelemans, G., Yungelson, L. R., Portegies Zwart, S. F., \& Verbunt, F. 2001, A\&A, 365, 491

Piro, A. L. 2011, ApJ, 740, L53

Pols, O. R., Schroder, K.-P., Hurley, J. R., Tout, C. A., \& Eggleton, P. P. 1998, MNRAS, 298, 525

Rodríguez-Gil, P., Santander-García, M., Knigge, C., et al. 2010, MNRAS, 407, L21

Rossiter, R. A. 1924, ApJ, 60, 15

Santander-García, M., Rodríguez-Gil, P., Jones, D., et al. 2011, in Asymmetric Planetary Nebulae 5 Conf., ed. A. A. Zijlstra, F. Lykou, I. McDonald, \& E. Lagadec, 259

Shporer, A., Brown, T., Mazeh, T., \& Zucker, S. 2011, arXiv:1107.4458

Shporer, A., Kaplan, D. L., Steinfadt, J. D. R., et al. 2010, ApJ, 725, L200

Sing, D. 2010, A\&A, 510, 21

Steinfadt, J. D. R., Kaplan, D. R., Shporer, A., Bildsten, L., \& Howell, S. B. 2010, ApJ, 716, L146

Sweigart, A. V., \& Mengel, J. G. 1979, ApJ, 229, 624

Taam, R., \& Sandquist, E. 2000, ARA\&A, 38, 113

Van Kerkwijk, M. H., Rappaport, S. A., Breton, R. P., et al. 2010, ApJ, 715, 51

Verbunt, F., \& Rappaport, S. A. 1988, ApJ, 332, 193

Wesson, R., Barlow, M. J., Corradi, R. L. M., et al. 2008, ApJ, 688, L21

Zucker, S., Mazeh, T., \& Alexander, T. 2007, ApJ, 670, 1326 